BULLETIN (New Series) OF THE

AMERICAN MATHEMATICAL SOCIETY

Volume 37, Number 1, Pages 25-38

S 0273-0979(99)00801-0

Article electronically published on December 21, 1999

\title{
THE PRESENT AND THE FUTURE OF MATHEMATICAL PHYSICS
}

\author{
PROFESSOR H. POINCARÉ
}

What is the present state of mathematical physics? What are its problems? What is its future? Is it about to change its orientation? Will the object and methods of this science appear in ten years to our immediate successors in the same light as they appear to us? Or are we to witness a far-reaching transformation? These are the questions we are forced to face to-day at the outset of our inquiry.

It is easy to ask; difficult to answer. If we felt tempted to hazard a prediction, we should easily resist this temptation by stopping to think of the nonsense the most eminent scholars of a hundred years ago would have spoken in answer to the question of what this science would be in the nineteenth century. They would have thought themselves bold in their predictions; and after the event how timid we should have found them! Do not expect of me therefore any kind of prophesy.

But if, like all prudent physicians, I refuse to give a prognosis, still I cannot deny myself a little diagnosis. Well, then, yes; there are symptoms of a serious crisis, which would seem to indicate that we may expect presently a transformation. However, there is no cause for great anxiety. We are assured that the patient will not die, and indeed we may hope that this crisis will be salutary, since the history of the past would seem to insure that. In fact, this crisis is not the first, and in order to understand it it is well to recall those which have gone before. Allow me a brief historical sketch.

Mathematical physics, as we are well aware, is an offspring of celestial mechanics, which gave it birth at the end of the eighteenth century at the moment when it had itself attained its complete development. The child, especially during its first years, showed a striking resemblance to its mother.

The astronomical universe consists of masses, undoubtedly of great magnitude, but separated by such immense distances that they appear to us as material points; these points attract each other in the inverse ratio of the squares of their distances, and this attraction is the only force which affects their motion. But if our senses were keen enough to show us all the details of the bodies which the physicist studies, the spectacle thus disclosed would hardly differ from the one which the astronomer contemplates. There too we should see material points separated by intervals which are enormous in comparison with their dimensions, and describing orbits according to regular laws. These infinitesimal stars are the atoms. Like the stars proper, they attract each other or repel, and this attraction or repulsion, which is along

2000 Mathematics Subject Classification. Primary 83A05, 70A05.

Reprinted from Bull. Amer. Math. Soc. 12 (1906), 240-260.

Translated with the author's permission by Professor J. W. Young.

Address delivered before the Section of Applied Mathematics of the International Congress of Arts and Science, St. Louis, September 24, 1904. 
the line joining them, depends only on the distance. The law according to which this force varies with the distance is perhaps not the law of Newton, but it is analogous thereto: instead of the exponent -2 we probably have another exponent, and from this diversity in the exponents proceeds all the diversity of the physical phenomena, the variety in qualities and sensations, all the world of color and sound which surrounds us; in a word, all nature.

Such is the primitive conception in its utmost purity. Nothing remains but to inquire in the different cases, what value must be given to this exponent in order to account for all the facts. On this model, for example, Laplace constructed his beautiful theory of capillarity; he simply regards the latter as a special case of attraction, or, as he says, of universal gravitation, and no one is surprised to find it in the middle of one of the five volumes of his celestial mechanics. More recently Briot believes he has laid bare the last secret of optics, when he has proved that the atoms of the ether attract each other in the inverse sixth power of the distance; and does not Maxwell, Maxwell himself, say somewhere that the atoms of a gas repel each other in the inverse ratio of the fifth power of the distance? We have the exponent -6 or -5 , instead of the exponent -2 ; but it is always an exponent.

Among the theories of this period there is a single one that forms an exception, namely that of Fourier; here there are indeed atoms acting at a distance; they send each other heat, but they do not attract each other, they do not stir. From this point of view, Fourier's theory must have appeared imperfect and provisional to the eyes of his contemporaries, and even to his own.

This conception was not without greatness; it was alluring, and many of us have not definitely given it up; they know that the ultimate elements of things will not be attained, except by disentangling with patience the complex skein furnished us by our senses; that progress should be made step by step without neglecting any intermediate portions; that our fathers were unwise in not wishing to stop at all the stations; but they believe that when we once arrive at these ultimate elements, we shall meet again the majestic simplicity of celestial mechanics.

Nor has this conception been useless; it has rendered us a priceless service inasmuch as it has contributed to making more precise the fundamental concept of the physical law. Let me explain: What did the ancients understand by a law? It was to them an internal harmony, statical as it were, and unchangeable; or else a model which nature tried to imitate. To us a law is no longer that at all; it is a constant relation between the phenomenon of to-day and that of to-morrow; in a word, it is a differential equation.

Here we have the ideal form of the physical law; and, indeed, it is Newton's law which first gave it this form. If, later on, this form has become inured in physics, it has become so precisely by copying as far as possible this law of Newton, by using celestial mechanics as a model.

Nevertheless there came a day when the conception of central forces appeared no longer to suffice, and this is the first of the crises to which I referred a moment ago.

What was done? Abandoned was the thought of exploring the details of the universe, of isolating the parts of this vast mechanism, of analyzing one by one the forces which set them going; and one was content to take as guides certain general principles which have precisely the object of relieving us of this minute study. How is this possible? Suppose we have before us any kind of machine; the part of the mechanism where the power is applied and the ultimate resultant motion alone are 
visible, while the transmissions, the intermediate gearing whereby the motion is communicated from one part to another, are hidden in the interior and escape our notice; we know not whether the transmissions are made by cog-wheels or by belts, by connecting-rods or other contrivances. Shall we say that it is impossible for us to learn anything about this machine unless we are allowed to take it apart? You well know that such is not the case, and that the principle of the conservation of energy suffices to furnish us the most interesting feature. We can easily show that the last wheel turns ten times more slowly than the first, since these two wheels are visible; and we can conclude therefrom that a couple applied to the first will be in equilibrium with a couple ten times as great applied to the second. To obtain this result, it is in no wise necessary to look into the mechanism of this equilibrium, or to know how the forces balance in the interior of the machine; it is sufficient to make sure that it is impossible for this balancing not to take place.

Very well! In the case of the universe, the principle of the conservation of energy can render us the same service. This universe also is a machine, much more complicated than any in use in the industries, of which nearly all the parts are deeply hidden; but by observing the motion of those which we can see, we can by the aid of this principle draw conclusions which will remain valid no matter what the details of the invisible mechanism which actuates them.

The principle of the conservation of energy, or Mayer's principle, is certainly the most important, but it is not the only one; there are others from which we can derive the same advantage. These are:

Carnot's principle, or the principle of the dissipation of energy.

Newton's principle, or the principle of the equality of action and reaction.

The principle of relativity, according to which the laws of physical phenomena must be the same for a stationary observer as for one carried along in a uniform motion of translation, so that we have no means, and can have none, of determining whether or not we are being carried along in such a motion.

The principle of the conservation of mass, or Lavoisier's principle. I will add the principle of least action.

The application of these five or six general principles to the various physical phenomena suffices to teach us what we may reasonably hope to know about them. The most remarkable example of this new mathematical physics is without doubt Maxwell's electro-magnetic theory of light. What is the ether? How are its molecules distributed? Do they attract or repel each other? Of these things we know nothing. But we know that this medium transmits both optical and electrical disturbances; we know that this transmission must take place in conformity with the general principles of mechanics and that suffices to establish the equations of the electro-magnetic field.

These principles are the boldly generalized results of experiment; but they appear to derive from their very generality a high degree of certainty. In fact, the greater the generality, the more frequent are the opportunities for verifying them, and such verifications, as they multiply, as they take the most varied and most unexpected forms, leave in the end no room for doubt.

Such is the second phase of the history of mathematical physics, and we have not yet left it. Shall we say that the first has been useless, that for fifty years science was on a wrong path and that there is nothing to do but to forget all that accumulation of effort which a vicious conception from the very beginning doomed to failure? By no means! Do you think the second period could have existed without the first? 
The hypothesis of central forces contained all the principles; it involved them as necessary consequences; it involved the principle of the conservation of energy, as well as that of mass, and the equality of action and reaction, and the law of least action, which appeared to be sure, not as experimental facts, but as theorems, and of which the statement had I know not how much greater precision and lesser generality than under their present form.

It is the mathematical physics of our fathers which has gradually made us familiar with these various principles, which has taught us to recognize them in the different garbs in which they are disguised. They have been compared with the results of experiment; it has been found necessary to change their expression in order to make them conform to the facts; thus they have been extended and strengthened. In this way they came to be regarded as experimental truths. The conception of central forces then became a useless support, or rather an encumbrance, inasmuch as it imposed upon the principles its own hypothetical character.

The bounds then are not broken, because they were elastic; but they have been extended. Our fathers who established them have not labored in vain; and in the science of to-day we recognize the general features of the outline they traced.

Are we now about to enter upon a third period? Are we on the eve of a second crisis? Are these principles on which we have reared everything about to fall in their turn? This has recently become a vital question.

Hearing me speak thus, you are thinking without doubt of radium, that great revolutionary of the present day; and indeed I shall return to it presently. But there is something else. It is not merely the conservation of energy that is concerned; all the other principles are in equal danger, as we shall see by successively passing them in review.

Let us begin with Carnot's principle. It is the only one which does not present itself as an immediate consequence of the hypothesis of central forces; quite to the contrary, indeed, it appears, if not actually to contradict this hypothesis, at least not to be reconcilable with it without some effort. If physical phenomena were due exclusively to the motion of atoms the mutual attractions of which depend only on the distance, it would seem that all these phenomena should be reversible; if all the initial velocities were reversed, these atoms, if still subject to the same forces, should traverse their trajectories in the opposite direction, just as the earth would describe backward this same elliptical orbit that it now describes forward, if the initial conditions of its motion had been reversed. Thus, if a physical phenomenon is possible, the inverse phenomenon should be equally possible, and one should be able to retrace the course of time. Now, it is not so in nature, and this it is precisely that the principle of Carnot teaches us; heat may pass from a hot body to a cold; it is impossible to compel it to take the opposite route and to reestablish differences of temperature which have disappeared. Motion can be entirely destroyed and transformed into heat by friction; the converse transformation can only occur partially.

Efforts have been made to reconcile this apparent contradiction. If the world tends toward uniformity, it is not because its ultimate parts, though diversified at the start, tend to become less and less different; it is because moving at random they become mixed. To an eye which could distinguish all the elements, the variety would remain always as great; every grain of this powder retains its originality and does not fashion itself after its neighbors; but as the mixture becomes more and 
more perfect, our rough senses perceive only uniformity. That is why, for example, temperatures tend to equalize themselves, without its being possible to go back.

A drop of wine, let us say, falls into a glass of water; whatever the internal motion of the liquid, we shall soon see it assume a uniformly roseate hue, and from then on no possible shaking of the vessel would seem to be capable of again separating the wine and the water. Here, then, we have what may be the type of the irreversible phenomenon of physics: to hide a grain of barley in a great mass of wheat would be easy; to find it again and to remove it is practically impossible. All this has been explained by Maxwell and Boltzmann, but the man who has put it most clearly was Gibbs, in a book too little read because it is a little difficult to read, in his Elements of Statistical Mechanics.

To those who take this point of view, Carnot's principle is an imperfect principle, a sort of concession to the frailty of our senses; it is because our eyes are too coarse that we do not distinguish the elements of the mixture; it is because our hands are too coarse that we cannot compel them to separate; the imaginary demon of Maxwell, who can pick out the molecules one by one, would be quite able to constrain the world to move backwards. That it should return of its own accord is not impossible; it is only infinitely improbable; the chances are that we should wait a long time for that combination of circumstances which would permit a retrogression; but, sooner or later, they will occur, after years, the number of which would require millions of figures. These reservations, however, all remained theoretical; they caused little uneasiness and Carnot's principle preserved all of its practical value.

But now here is where the scene changes. The biologist, armed with his microscope, has for a long time noticed in his preparations certain irregular motions of small particles in suspension; this is known as Brown's motion. He believed at first that it was a phenomenon of life, but he soon saw that inanimate bodies hopped about with no less ardor than others; he then turned the matter over to the physicists. Unfortunately, the physicists did not become interested in the question for a long time. Light is concentrated, so they argued, in order to illuminate the microscopical preparation; light involves heat, and this causes differences in temperature and these produce internal currents in the liquid, which bring about the motions referred to.

M. Gouy had the idea of looking a little more closely, and thought he saw that this explanation was untenable; that the motion becomes more active as the particles become smaller, but that they are uninfluenced by the manner of lighting. If, then, these motions do not cease, or, rather, if they come into existence incessantly, without borrowing from any external source of energy, what must we think? We must surely not abandon on this account the conservation of energy; but we see before our eyes motion transformed into heat by friction and conversely heat changing into motion, and all without any sort of loss, since the motion continues forever. It is the contradiction of Carnot's principle. If such is the case, we need no longer the infinitely keen eye of Maxwell's demon in order to see the world move backward; our microscope suffices. The larger bodies, those of a tenth of a millimeter, for example, are bombarded from all sides by the moving atoms, but they do not stir, because these shocks are so numerous that the law of probabilities requires them to compensate each other; but the smaller particles are hit too rarely to have this compensation take place with any degree of certainty and are thus incessantly tossed about. And so one of our principles is already in danger. 
Let us consider the principle of relativity; this principle is not only confirmed by our daily experience, not only is it the necessary consequence of the hypothesis of central forces, but it appeals to our common sense with irresistible force. And yet it also is being fiercely attacked. Let us think of two electrified bodies; although they seem to be at rest, they are, both of them, carried along with the motion of the earth; Rowland has shown us that an electric charge in motion is equivalent to a current; these two charged bodies, then, are equivalent to two parallel currents in the same direction; these two currents should attract each other. By measuring this attraction we should be measuring the velocity of the earth; not its velocity relative to the sun and stars, but its absolute velocity.

I know what will be said; it is not its absolute velocity; it is its velocity relative to the ether. But, how unsatisfactory that is! Is it not clear that with this interpretation, nothing could be inferred from the principle? It could no longer teach us anything, simply because it would no longer fear any contradiction. Whenever we have succeeded in measuring anything, we would always be free to say that it is not the absolute velocity, and if it is not the velocity relative to the ether, it might always be the velocity relative to some new unknown fluid with which we might fill all space.

And then experiment, too, has taken upon itself to refute this interpretation of the principle of relativity; all the attempts to measure the velocity of the earth relative to the ether have led to negative results. Herein experimental physics has been more faithful to the principle than mathematical physics; the theorists would have dispensed with it readily in order to harmonize the other general points of view; but experimentation has insisted on confirming it. Methods were diversified; finally Michelson carried precision to its utmost limits; nothing came of it. It is precisely to overcome this stubborness that to-day mathematicians are forced to employ all their ingenuity.

Their task was not easy, and if Lorentz has succeeded, it is only by an accumulation of hypotheses.

The most ingenious idea is that of local time. Let us imagine two observers, who wish to regulate their watches by means of optical signals; they exchange signals, but as they know that the transmission of light is not instantaneous, they are careful to cross them. When station B sees the signal from station A, its timepiece should not mark the same hour as that of station $\mathrm{A}$ at the moment the signal was sent, but this hour increased by a constant representing the time of transmission. Let us suppose, for example, that station A sends it signal at the moment when its time-piece marks the hour zero, and that station B receives it when its time-piece marks the hour $t$. The watches will be set, if the time $t$ is the time of transmission, and in order to verify it, station B in turn sends a signal at the instant when its time-piece is at zero; station A must then see it when its time-piece is at $t$. Then the watches are regulated.

And, indeed, they mark the same hour at the same physical instant, but under one condition, namely, that the two stations are stationary. Otherwise, the time of transmission will not be the same in the two directions, since the station $A$, for example, goes to meet the disturbance emanating from $B$, whereas station $B$ flees before the disturbance emanating from $A$. Watches regulated in this way, therefore, will not mark the true time; they will mark what might be called the local time, so that one will gain on the other. It matters little, since we have no means of perceiving it. All the phenomena which take place at $A$, for example, will be 
behind time, but all just the same amount, and the observer will not notice it since his watch is also behind time; thus, in accordance with the principle of relativity he will have no means of ascertaining whether he is at rest or in absolute motion. Unfortunately this is not sufficient; additional hypotheses are necessary. We must admit that the moving bodies undergo a uniform contraction in the direction of the motion. One of the diameters of the earth, for example, is shortened by $1 / 200000000$ as a result of our planet's motion, whereas the other diameter preserves its normal length. Thus we find the last minute differences accounted for. Then there is still the hypothesis concerning the forces. Forces, whatever their origin, weight as well as elasticity, will be reduced in a certain ratio in a world endowed with a uniform translatory motion; or rather that would happen for the components at right angles to the direction of translation; the parallel components will not change. Let us then return to our example of the two electrified bodies; they repel each other; but at the same time, if everything is carried along in a uniform transition, they are equivalent to two parallel currents in the same direction, which attract each other.

This electrodynamic attraction is, then, subtracted from the electrostatic repulsion, and the resultant repulsion is weaker than if the two bodies had been at rest. But since we must, in order to measure this repulsion, balance it by another force, and since all these other forces are reduced in the same ratio, we observe nothing. Everything, then, appears to be in order. But have all doubts been dissipated? What would happen if we could communicate by signals other than those of light, the velocity of propagation of which differed from that of light? If, after having regulated our watches by the optimal method, we wished to verify the result by means of these new signals, we should observe discrepances due to the common translatory motion of the two stations. And are such signals inconceivable, if we take the view of Laplace, that universal gravitation is transmitted with a velocity a million times as great as that of light?

Thus the principle of relativity has in recent times been valiantly defended; but the very vigor of the defense shows how serious was the attack.

And now let us speak of the principle of Newton, concerning the equality of action and reaction. This principle is intimately connected with the preceding and it would seem that the fall of one would involve the fall of the other. Nor must we be surprised to find here again the same difficulties.

The electrical phenomena, it is thought, are due to displacements of small charged particles called electrons which are immersed in the medium we call ether. The motions of these electrons produce disturbances in the surrounding ether; these disturbances are propagated in all directions with the velocity of light, and other electrons initially at rest are displaced when the disturbance reaches the portions of the ether in which they lie. The electrons, then, act one upon the other, but this action is not direct; it takes place by mediation of the ether. Under these conditions, is it possible to have equality between action and reaction, at least for an observer who takes account only of the motion of matter, that is of the electrons, and who ignores that of the ether which he is unable to see? Evidently not. Even if the compensation were exact, it could not be instantaneous. The disturbance is propagated with a finite velocity; it reaches the second electron, therefore, only after the first has long been reduced to rest. This second electron will, then, after an interval, be subjected to the action of the first, but will certainly not at that moment react upon it, since there is no longer anything in the neighborhood of this first electron that stirs. 
The analysis of the facts will allow us to become more definite. Let us imagine, for example, a Hertzian oscillator such as those used in wireless telegraphy; it sends energy in all directions; but we may attach to it a parabolic mirror, as was done by Hertz with his smallest oscillators, so as to send all the energy produced in a single direction. What then will happen according to the theory? Why, the apparatus will recoil as though it were a cannon and the projected energy a ball, and that contradicts the principle of Newton, since our present projectile has no mass; it is not matter, it is energy. It is the same, moreover, in the case of a light-house having a reflector, since light is merely a disturbance in the electro-magnetic field. This light-house would recoil, as though the light it sends forth were a projectile. What is the force that must produce this recoil? It is what is known as the MaxwellBartholdi pressure; it is very small, and to put it in evidence caused much trouble, even with the most sensitive radiometers; but it is sufficient for our purpose that it exists.

If all the energy issuing from our oscillator strikes a receiver, the latter will act as though it had received a physical shock, which in a sense will represent the compensation of the oscillator's recoil; the reaction will be equal to the action, but they will not be simultaneous; the receiver will advance, but not at the instant when the oscillator recoils. If the energy is propagated indefinitely without meeting a receiver, the compensation will never take place.

Shall we say that the space which separates the oscillator from the receiver and which the disturbance must traverse in passing from one to the other, is not empty, but is filled not only with ether, but with air, or even in inter-planetary space with some subtile, yet ponderable fluid; that this matter receives the shock, as does the receiver, at the moment the energy reaches it, and recoils, when the disturbance leaves it? That would save Newton's principle, but it is not true. If the energy during its propagation remained always attached to some material substratum, this matter would carry the light along with it and Fizeau has shown, at least for the air, that there is nothing of the kind. Michelson and Morley have since confirmed this. We might also suppose that the motions of matter proper were exactly compensated by those of the ether; but that would lead us to the same considerations as those made a moment ago. The principle, if thus interpreted, could explain anything, since whatever the visible motions we could imagine hypothetical motions to compensate them. But if it can explain anything, it will allow us to foretell nothing; it will not allow us to choose between the various possible hypotheses, since it explains everything in advance. It therefore becomes useless.

And then the suppositions that must be made concerning the motions of the ether are not very satisfactory. If the electric charges were doubled, it would be natural to suppose that the velocities of the atoms of the ether also became twice as great, and for the compensation it would be necessary that the mean velocity of the ether become four times as great.

This is why I have for a long time thought that these consequences of the theory, which contradict Newton's principle, would some day be abandoned; and yet the recent experiments on the motion of the electrons emitted by radium seem rather to confirm them.

I now come to Lavoisier's principle concerning the conservation of mass. This is certainly a principle which cannot be tampered with without shaking the science of mechanics. And still there are persons who think that it seems true to us only because in mechanics we consider only moderate velocities, and that it would cease 
to be so for bodies having velocities comparable with that of light. Now, such velocities are at present believed to have been realized; the cathode rays and those of radium would seem to be formed of very minute particles or electrons that move with velocities that are no doubt less than that of light, but which appear to be about one tenth or one third of it.

These rays can be deflected either by an electric or by a magnetic field, and by comparing these deflections it is possible to measure both the velocity of the electrons and their mass (or rather the ratio of their mass to their charge). But it was found that as soon as these velocities approached that of light a correction was necessary. Since these particles are electrified, they cannot be displaced without disturbing the ether; to put them in motion, it is necessary to overcome a double inertia, that of the particle itself and that of the ether. The total or apparent mass that is measured is then composed of two parts: the real or mechanical mass of the particle and the electrodynamic mass representing the inertia of the ether.

Now, the calculations of Abraham and the experiments of Kaufmann have shown that the mechanical mass properly so called is nothing, and that the mass of the electrons, at least of the negative electrons, is purely of electrodynamic origin. This is what compels us to change our definition of mass; we can no longer distinguish between the mechanical mass and the electrodynamic mass, because then the first would have to vanish; there is no other mass than the electrodynamic inertia; but in this case, the mass can no longer be constant; it increases with the velocity; and indeed it depends on the direction, and a body having a considerable velocity will not oppose the same inertia to forces tending to turn it off its path that it opposes to those tending to accelerate or retard its motion.

There is indeed another resource: the ultimate elements of bodies are electrons, some with a negative charge, others with a positive charge. It is understood that the negative electrons have no mass; but the positive electrons, from what little is known of them, would seem to be much larger. They perhaps have besides their electrodynamic mass a true mechanical mass. The real mass of a body would then be the sum of the mechanical masses of its positive electrons, the negative electrons would not count; the mass defined in this way might still be constant.

Alas, this resource is also denied. Let us recall what we said concerning the principle of relativity and the efforts made to save it. And it is not only a principle that is to be saved; the indubitable results of Michelson's experiments are involved. And so, as was above seen, Lorentz, to account for these results, was obliged to suppose that all forces, whatever their origin, are reduced in the same ratio in a medium having a uniform translatory motion. But that is not sufficient; it is not enough that this should take place for the real forces, it must also be the same in the case of the forces of inertia; it is necessary, therefore - so he says - that the masses of all particles be influenced by a translation in the same degree as the electromagnetic masses of the electrons.

Hence, the mechanical masses must vary according to the same laws as the electrodynamic; they can then not be constant.

Do I need to remark that the fall of Lavoisier's principle carries with it that of Newton's? The latter implies that the center of gravity of an isolated system moves in a straight line; but if there no longer exists a constant mass, there no longer exists a center of gravity; indeed the phrase would be meaningless. This is why I said above that the experiments on cathode rays seemed to justify the doubts of Lorentz concerning Newton's principle. 
From all these results, if they were to be confirmed, would issue a wholly new mechanics which would be characterized above all by this fact, that there could be no velocity greater than that of light, ${ }^{1}$ any more than a temperature below that of absolute zero. For an observer, participating himself in a motion of translation of which he has no suspicion, no apparent velocity could surpass that of light, and this would be a contradiction, unless one recalls the fact that this observer does not use the same sort of timepiece as that used by a stationary observer, but rather a watch giving the "local time."

Here we are then face to face with a question, of which I shall confine myself to the mere statement. If there is no longer any mass what becomes of Newton's law?

Mass has two aspects: it is at the same time a coefficient of inertia and an attracting mass entering as a factor into Newton's law of attraction. If the coefficient of inertia is not constant, can the attracting mass be constant? This is the question.

The principle of the conservation of energy at least still remained and appeared more finely established. Shall I recall to your minds how it too was thrown into discredit? That event made more noise than the preceding; the journals are full of it. Ever since the first work of Becquerel, and above all after the Curies had discovered radium, it was seen that every radioactive substance was an inexhaustible source of radiation. Its activity seemed to continue without change through months and years. That is already a strain on the principles; these radiations in fact were energy, and from the same piece of radium came forth this energy and it came forth indefinitely. But these quantities of energy were too minute to be measured; at least that was the belief, and the matter caused little uneasiness.

The scene changed when Curie thought of placing the radium in a calorimeter. It was then seen that the quantity of heat continuously generated was very considerable.

The explanations advanced were numerous; but in a case of this kind it is not possible to say that an abundance of good does no harm: as long as one explanation has not displaced the others we can not be sure that any one of them is good. For some time, however, one of these explanations seems to be gaining the upper hand and we may reasonably hope that we hold the key to the mystery.

Sir W. Ramsey has attempted to show that radium is transformed, that it contains an enormous amount of energy, but not an inexhaustible amount. The transformation of radium must then produce a million times as much heat as any known transformation; the radium would be exhausted in 1250 years; that is not long, but you see that we are at least sure of being bound to the present state of affairs for some hundreds of years. While we wait our doubts subsist.

In the midst of such ruin, what remains standing? The principle of least action up to now is intact, and Larmor appears to think that it will long survive the others. It is in fact more vague and even more general.

In the presence of this general collapse of principles, what attitude should mathematical physics take? First of all, before becoming too excited, it is well to ask whether all this is really true. All this disparagement of principles is encountered only in the case of the infinitely small; the microscope is needed to see Brown's motion, the electrons are rather tiny, radium is very rare and never more than a few milligrams are together; and then we can ask whether by the side of the minute

\footnotetext{
${ }^{1}$ Because bodies would oppose an increasing inertia to the causes that would tend to accelerate their motion; and when approaching the velocity of light, this inertia would become infinite.
} 
thing that was observed, there was not another minute thing which was not noticed and which counterbalanced the first.

The question is surely debatable, and apparently only experiment can solve it. We should merely have to turn the matter over to the experimenters and, while waiting for them definitely to settle the controversy, not to trouble ourselves with these disquieting problems, and to keep quietly at our work, as though the principles were still unchallenged. We certainly have enough to do without leaving the domain where they can be applied with all certainty; we have enough to keep us busy during this period of doubt.

And yet is it really true that we can do nothing to relieve science of these doubts? It must indeed be said that it has not been experimental physics alone that has brought them into existence; mathematical physics has contributed its share. It was the experimenters who saw radium emit energy; but the theorists were the ones who brought to light all the difficulties inherent in the propagation of light through a moving medium; had it not been for them, they probably would not have been noticed. They have, then, done their best to embarrass us; it is no more than just that they should help us to extricate ourselves.

They must subject to a searching criticism all the new conceptions that I have outlined to-day, nor must they abandon the principles except after a loyal effort to save them. What can they do in this direction? That is what I shall seek to explain.

Among the most interesting problems of mathematical physics a place should be set apart for those that belong to the kinetic theory of gases.

Much has already been done toward their solution, but much remains to do. This theory is an everlasting paradox. We have reversibility in the premises and irreversibility in the conclusions, and a deep chasm between the two. Will statistical considerations, and the law of large numbers, suffice to fill it up? Many points still remain obscure to which it will be necessary to return, and that without doubt several times. In clearing them up, the meaning of Carnot's principle will be better understood, and its general position in dynamics; and we shall be better able to interpret the curious experiment of Gouy to which I referred above.

Should we not also make an effort to obtain a more satisfactory theory of the electrodynamics of moving bodies? It is here, above all, as I indicated sufficiently a short time ago, that the difficulties accumulate; even though we heap up hypotheses, we can not satisfy all the principles at once; no one has succeeded so far in saving some without sacrificing others. But all hope of obtaining better results is not yet lost. Let us, then, take the theory of Lorentz. Let us turn it over and over, let us modify it little by little, and all will be well, perhaps.

Indeed, instead of supposing that bodies in motion undergo a contraction in the direction of motion and that this contraction is the same whatever the nature of these bodies and the forces to which they are subjected, could not a simpler and more natural hypothesis be made? One might suppose, for example, that it is the ether which changes when it is in relative motion with respect to the material substance which passes through it; that, when thus modified, it no longer transmits the disturbances with the same velocity in all directions. It would transmit more rapidly those disturbances which are being propagated parallel to the motion of the substance, be it in the same direction or in the opposite, and less rapidly those which are propagated at right angles. The wave surfaces would then no longer be 
spheres, but ellipsoids, and one could do without this extraordinary contraction of all bodies.

I am giving this only by way of example, since the modifications which could be tried are evidently susceptible of infinite variation.

It is possible also that astronomy may some day furnish us with data on this point: she it was, in fact, who raised the question by making known to us the phenomenon of the aberration of light. If the theory of the aberration of light is roughly constructed, a curious result is arrived at. The apparent positions of the stars differ from their real positions by reason of the earth's motion, and since this motion is variable the apparent positions vary. The real position we are unable to ascertain, but we can observe the variations of the apparent position. Observations on aberration then show us, not the earth's motion, but the variations of this motion. They can, therefore, teach us nothing concerning the absolute motion of the earth.

These at any rate are the facts under a first approximation; but such would no longer be the case if we could observe the thousandth part of a second. It would then be seen that the variation in the apparent motion of the star depends not only on the variation in the earth's motion, a variation which is well known, since it is the motion of our globe in its elliptical orbit, but also on the mean value of this motion, so that the constant of aberration would not be quite the same for all stars, and that the differences would make known to us the earth's absolute motion in space.

This would be, in another form, the end of the principle of relativity. We are far, it is true, from being able to observe the thousandth part of a second, but after all, say some, the earth's total absolute velocity is perhaps much greater than its velocity relative to the sun; if it were for instance $300 \mathrm{~km}$. per second, instead of $30 \mathrm{~km}$., that would suffice to make the phenomenon observable.

I believe that by reasoning in this manner we carry simplicity in the theory of aberration too far. Michelson has shown, as I have said, that the methods of physics are powerless to put absolute motion in evidence; I am convinced that in the case of astronomical methods it will be the same, no matter how far precision may be carried.

However that may be, the data which astronomy will furnish in this direction will one day be valuable to the physicist. In the meantime I believe that the theorists, keeping in mind the experiments of Michelson, may count on a negative result, and that they would do useful work by constructing a theory of aberration which takes account of it in advance.

But let us return to the earth. There, too, we can help the experimenters. We can, for example, prepare the way by studying thoroughly the dynamics of the electrons; not, be it well understood, by staring from a single hypothesis, but by multiplying the hypotheses as much as possible. It would then be the part of the physicist to use our work in searching for the crucial experiment which would decide between them.

This dynamics of the electrons can be approached from many sides; but among the roads that lead there, there is one which has been somewhat neglected, and yet it is one of those that promise us the most surprises. It is the motion of electrons that produces the lines of the spectrum; this is proved by the phenomenon of Zeemann; what vibrates in an incandescent body is affected by a magnet, and is hence electrified. This is a first very important point; but no one has gone into 
the question any further. Why are the lines of the spectrum distributed according to a regular law? These laws have been studied by the experimenters in the greatest detail; they are very precise and comparatively simple. A first study of these arrangements brings to mind the harmonics encountered in acoustics; but the difference is great. Not only are the numbers of vibrations not successive multiples of a single number, but we even find nothing analogous to the roots of those transcendental equations, to which we are led by so many problems of mathematical physics: that of the vibrations of an elastic body of any shape, of the Hertzian oscillations in a generator of any form, the problem of Fourier on the cooling of a solid body.

The laws are simpler, but they are of an entirely different kind. To mention only one of these differences, for harmonics of high order the number of vibrations tends toward a finite limit, instead of increasing indefinitely.

This has not yet been explained, and I believe that here is one of the most important of nature's secrets. Lindemann has made a praiseworthy attempt, but in my opinion without success. This attempt should be renewed. We shall thus penetrate, so to speak, into the intimacies of matter. And, from the particular point of view that we occupy to-day, when we shall know why the vibrations of incandescent bodies differ in this way from the vibrations of ordinary elastic bodies, why the electrons do not behave like the matter with which we are familiar, we shall better understand the dynamics of the electrons and it will perhaps be easier for us to reconcile them with the principles.

Now suppose that all these efforts should fail (and when all is said, I do not believe they will), what should be done? Should we seek to rebuild these shattered principles by one stroke, as it were? That, evidently, is always possible, and I take back nothing of what I once said. "Did you not write," you might say, if you were seeking a quarrel with me, "did you not write that the principles, though they are of experimental origin, are now beyond the possibility of experimental attack, because they have become conventions? And now you come to tell us that the triumphs of the most recent experiments put these principles in danger."

Very well, I was right formerly, and I am not wrong to-day. I was right formerly, and what is taking place at present is another proof of it. Let us take, for example, the calorimetric experiment of Curie with radium. Is it possible to reconcile it with the principle of the conservation of energy? This has been attempted in many ways; but there is one among them to which I wish to call your attention; it is not the explanation which is tending to-day to prevail, but it is one of those that have been suggested. Radium is assumed to be only an intermediary, merely to store radiations of an unknown nature that fly through space in all directions, traversing all bodies except radium without being changed by this passage, and without exerting on them any action whatever. Radium alone can appropriate a little of their energy and then return it to us in various forms.

How useful this explanation is and how convenient! In the first place it is nonverifiable and hence irrefutable. Then, it can serve to account for any contradiction to Mayer's principle; it answers in advance not only the objection of Curie, but all other objections that the experimenters of the future may accumulate. This new and unknown energy could be used for anything.

That is exactly what I said, and by such means it is easy to show that our principle is safe from experimental attacks. 
But then, what have we gained by this stroke? The principle is intact, but henceforth what is it good for? It enabled us to foresee that under such and such conditions we could count on a certain amount of energy; it imposed a limit; but now that there has been placed at our disposal this indefinite supply of new energy, we are no longer limited by anything; and, as I have also written, if a principle ceases to be productive, experiment, without contradicting it directly, would nevertheless condemn it.

That, then, is not what should be done. We should have to rebuild from the beginning. If we were driven to this necessity, we could easily console ourselves. We should not be obliged to conclude that science can never do aught but the work of a Penelope, that it can only raise ephemeral structures which it is soon forced to demolish completely with its own hands.

As I have said, we have already passed through a similar crisis. I have shown you that in the second mathematical physics, that of general principles, one finds traces of the first, that of central forces; it will be the same if we are to know a third. Just so with the animal that casts its outer shell, that bursts the skin that has become too small and grows a new one; under the new covering can always be recognized the essential traits of the organism that survives.

In what direction we are going to expand we are unable to foresee. Perhaps it is the kinetic theory of gases that will forge ahead and serve as a model for the others. In that case, the facts that appeared simple to us at first will be nothing more than the resultants of a very large number of elementary facts which the laws of probability alone would induce to work toward the same end. A physical law would then assume an entirely new aspect; it would no longer be merely a differential equation, it would assume the character of a statistical law.

Perhaps too we shall have to construct an entirely new mechanics, which we can only just get a glimpse of, where, the inertia increasing with the velocity, the velocity of light would be a limit beyond which it would be impossible to go. The ordinary, simpler mechanics would remain a first approximation since it would be valid for velocities that are not too great, so that the old dynamics would be found in the new. We should have no reason to regret that we believed in the older principles, and indeed since the velocities that are too great for the old formulas will always be exceptional, the safest thing to do in practice would be to act as though we continued to believe in them. They are so useful that a place should be saved for them. To wish to banish them altogether would be to deprive oneself of a valuable weapon. I hasten to say, in closing, that we are not yet at that pass, and that nothing proves as yet that they will not come out of the fray victorious and intact. 